\title{
Transient endothelial injury and release of lupus anticoagulant in COVID-19
}

\author{
Antonin Trimaille ${ }^{1,2}$ (1) $\cdot$ Benjamin Marchandot ${ }^{1} \cdot$ Walid Oulehri $^{3} \cdot$ Adrien Carmona $^{1} \cdot$ Olivier Vollmer $^{4}$. \\ Vincent Poindron ${ }^{4} \cdot$ Kensuke Matsushita $^{1,2} \cdot$ Laurent Sattler $^{5} \cdot$ Lelia Grunebaum $^{5} \cdot$ Anne-Sophie Korganow $^{4}$. \\ Valerie Schini-Kerth ${ }^{2} \cdot$ Olivier Morel $^{1,2}$
}

Accepted: 18 May 2021 / Published online: 28 May 2021

(c) The Author(s), under exclusive licence to Springer Science+Business Media, LLC, part of Springer Nature 2021

Coronavirus disease 2019 (COVID-19) has been described as an endothelial disease associated with a procoagulant state and a high prevalence of lupus anticoagulant (LA) $[1,2]$. While the association between LA and thrombosis remains controversial [3], a recent study showed that markers of endothelial injury predict mortality during the acute phase [4]. No study has so far evaluated the persistence of endothelial injury after recovery. Here, we report the results of a systematic biologic assessment more than 12 weeks after the acute phase of COVID-19.

Patients hospitalized for COVID-19 at Strasbourg University Hospital, France, and tested positive for LA were included in the Microparticles in COVID-19 (MICO) study. COVID-19 was confirmed by a positive result of a reversetranscriptase-polymerase-chain-reaction (RT-PCR) assay of a specimen collected on a nasopharyngeal swab according to the World Health Organization (WHO) guidance. Patients with findings typical of COVID-19 at chest computed tomography (CT), (i.e. bilateral and peripheral ground glass opacities and/or alveolar consolidations) and for whom COVID-19 testing was either inconclusive or could not be

Olivier Morel

olivier.morel@chru-strasbourg.fr

1 Department of Cardiovascular Medicine, Nouvel Hôpital Civil, Strasbourg University Hospital, 1 place de l'Hôpital, 67000 Strasbourg, France

2 INSERM (French National Institute of Health and Medical Research), UMR 1260, Regenerative Nanomedicine, FMTS, Strasbourg, France

3 Department of Intensive Care Medicine, Strasbourg University Hospital, Strasbourg, France

4 Department of Immunology, Strasbourg University Hospital, Strasbourg, France

5 Department of Hemostasis, Strasbourg University Hospital, Strasbourg, France performed, were considered as confirmed COVID-19 cases by a multidisciplinary team. Medical management was left at the discretion of the treating physician. When decided, thromboprophylaxis was achieved with Enoxaparin at 4000 international units (IU)/24 h, Fondaparinux at $2.5 \mathrm{mg} / 24 \mathrm{~h}$ or unfractionated heparin at $4800 \mathrm{IU} / 24 \mathrm{~h}$ (standard preventive treatment), with Enoxaparin at $4000 \mathrm{IU}$ twice $/ 24 \mathrm{~h}$ (reinforced preventive treatment) or with Enoxaparin $100 \mathrm{IU} / \mathrm{kg}$ twice $/ 24 \mathrm{~h}$ (therapeutic).

Blood samples were collected in $0.109 \mathrm{M}$ sodium citrate tubes during acute phase and at least 12 weeks after COVID19 diagnosis. Fibrinogen, activated partial thromboplastin time (aPTT), D-dimer and von Willebrand factor antigen (vWF:Ag) were determined in plasma immediately after a single centrifugation ( $2500 \mathrm{~g}$ for $10 \mathrm{~min}$ ). Assays were delayed for LA detection. Platelet-poor plasma (PPP) was obtained by double centrifugation $(2 \times 2500 \times g$ for $10 \mathrm{~min})$, then frozen at $-80{ }^{\circ} \mathrm{C}$ until use. PPP was thawed for 4 min in a $37^{\circ} \mathrm{C}$ water bath just before the assays. All haemostasis assays (fibrinogen, aPTT, D-Dimer, vWF:Ag, LA detection) were analysed on STA-R® Evolution (Diagnostica Stago $\circledR$, Asnières-sur-Seine, France) with standard commercial reagents and protocols. LA detection was based on several tests. First, two screening tests were performed, respectively a Diluted Russel Viper Venom Time (dRVVT screen) made with the STA®-Staclot dRVV Screen reagent (Stago), and an aPTT performed with the STA®-PPT A reagent (Stago). Positivity of one or both screening tests induced a mixing test at 1:1 proportion with a commercial frozen PNP (Cryocheck $^{\mathrm{TM}}$ Pooled Normal Plasma, Cryocheck, Montpellier, France). Moreover, a positive dRVVT screen induced a confirmatory test with an increased concentration of phospholipids (dRVVT confirm), performed with the STA®Staclot dRVV Confirm reagent (Stago). dRVVT screen, DRVVT confirm and aPTT results were expressed as a ratio of patient-to-PNP. Mixing tests results were expressed as an 
index of circulating anticoagulant (ICA). LA was considered as positive only if the normalized dRVVT ratio (screen ratio/ confirm ratio) was $>1.2$ and all causes of false positive were excluded (i.e. anticoagulation conditions).

The study was approved by the national agency for the safety of medicines (Number 2020-A01500-39). Written informed consent was obtained from all study participants.

LA assays were performed in 262 patients hospitalized for COVID-19 in 10 departments of Strasbourg University Hospital, France between March 3 and May 5, 2020 and 56 had positive LA. Five patients were excluded from the analysis because of direct oral anticoagulant treatment at the time of follow-up. A total of 51 patients were included in the final analysis. The main results are shown in the Table 1 . The mean age was 61 years and 39 patients $(76.5 \%)$ were male.

During the acute phase of COVID-19, 38 patients (74.5\%) required mechanical ventilation and 10 patients (19.7\%) presented a venous thrombotic event. Mean von Willebrand factor antigen (vWF:Ag) level was 409.5\%. As previously mentioned, all patients were positive for LA.

Follow-up visit was performed at a median of 144 (interquartile range 129-179) days after COVID-19 diagnosis. LA detection was positive only in three patients (5.9\%) and mean level of vWF:Ag was $158.0 \%$ at the time of followup (Fig. 1). Clinical and biological characteristics of these three patients are described in the Supplementary Table I. Of note, two young male patients required mechanic ventilation at the acute phase and none of the three had venous thromboembolism.

Acute COVID-19 is characterized by an endothelial injury secondary to endothelial cells viral invasion and cytokine storm [1]. As admitted in antiphospholipid syndrome pathophysiology [5], our work suggest that the subsequent plasma membrane remodeling is susceptible to expose intracellular phospholipid to extracellular environment leading to LA formation. In addition, the loss of endothelium antithrombotic effects associated with coagulation abnormalities may expose patients to a higher risk of thrombosis $[1,6,7]$. This pathophysiologic hypothesis is consistent with the increased levels of vWF:Ag in our cohort and the high frequency of venous thrombosis at the acute phase.

As described in other viral diseases [8], we showed disappearance of LA in a large majority of patients. In addition, the drastic decrease of vWF:Ag levels suggests an endothelial injury recovery at distance from acute COVID-19, clinically translated by the absence of thrombosis event during
Table 1 Main characteristics of the study population $(\mathrm{n}=51)$

\begin{tabular}{lll}
\hline Characteristics & $\begin{array}{l}\text { Value } \\
\text { Acute phase }\end{array}$ & Follow-up \\
\hline Demographics & & \\
Age-years & $61 \pm 13$ & \\
Male-n (\%) & $39(76.5)$ & \\
Body mass index-kg/m ${ }^{2}$ & $27.3 \pm 3.8$ & \\
Cardiovascular risk factors-n $(\%)$ & & \\
Hypertension & $24(47.1)$ & \\
Dyslipidemia & $15(29.4)$ & \\
Diabetes & $7(13.7)$ & \\
Smoking & $2(3.9)$ & \\
Comorbidity & & \\
Coronary artery disease & $5(9.8)$ & \\
Heart failure & $1(2.0)$ & \\
Atrial fibrillation & $1(2.0)$ & \\
Venous thromboembolism & $3(5.9)$ & \\
Chronic respiratory disease & $6(11.8)$ & \\
History of cancer & $4(7.8)$ & $0(0.0)$ \\
COVID-19 outcomes & & \\
Venous thromboembolism & $10(19.6)$ & $0(0.0)$ \\
Acute pulmonary embolism & $8(15.7)$ & \\
Deep vein thrombosis & $3(5.9)$ & \\
Transfer to intensive care unit & $41(80.4)$ & \\
Mechanic ventilation & $38(74.5)$ & \\
Laboratory findings* & & \\
CRP-mg/L & $175.6 \pm 10.0)$ \\
Fibrinogen-g/L & $7.5 \pm 1.6$ & \\
aPTT-\% & $1.4 \pm 0.5$ & \\
D-Dimer-ng/mL & $3841 \pm 4375.9$ \\
LA detection-n (\%) & $51(100)$ & \\
vWF:Ag-\% & \\
\hline
\end{tabular}

Data are presented as mean \pm standard deviation in case of any other indication

*Blood samples were obtained at a median time of 144 days after COVID-19 diagnosis

${ }^{\dagger} \mathrm{VWF}$ :Ag level was measured in 25 patients at acute phase of COVID-19 and in all patients at follow-up

aPTT activated partial thromboplastin time, COVID-19 coronavirus disease 2019, CRP C reactive protein, $L A$ lupus anticoagulant, vWF:Ag, von Willebrand factor antigen

the follow-up. Although our observational data do not prove causation, they suggest that endothelial dysfunction is transient in COVID-19 patients and therefore associated to a potential temporary and limited pathophysiological effect. 
A

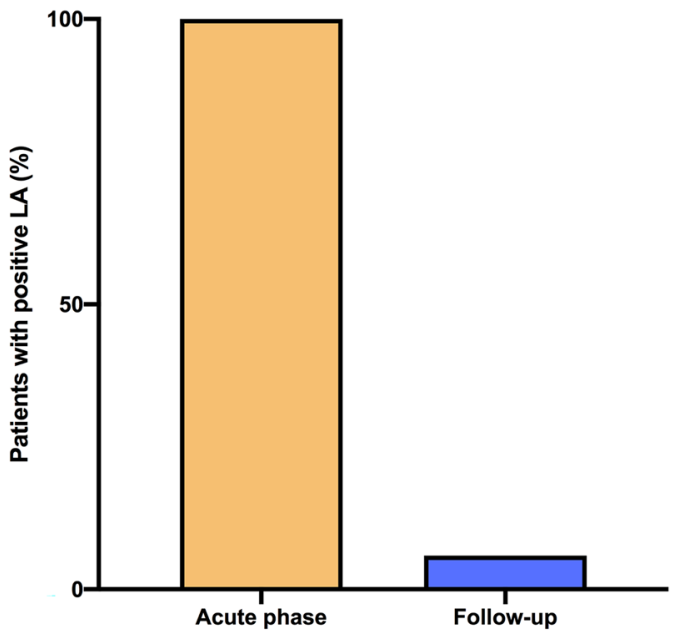

Fig. 1 Transient lupus anticoagulant positivity and high vWF:Ag levels during acute COVID-19. The Figure shows the appearance of positive lupus anticoagulant (Panel A) and higher vWF:Ag levels (Panel B) at acute phase of COVID-19 as compared to the recovery

Supplementary Information The online version contains supplementary material available at https://doi.org/10.1007/s11239-021-02485-5.

Acknowledgements The authors would like to formally acknowledge the commitment of all front-line healthcare workers during the COVID-19 crisis in the University Hospital of Strasbourg as they commit themselves to difficult work and also put themselves at risk of infection. Strasbourg's University Hospital is proud of the commitment of all nurses, including newly graduated nurses, medical students, health support staff and volunteers.

Funding This work was supported by GERCA (Groupe pour l'Enseignement, la prévention et la Recherche Cardiologique en Alsace) and Fondation Cœur et Recherche.

\section{Declarations}

Conflict of interest The author declares that they have no conflict of interest.

\section{References}

1. Libby P, Lüscher T (2020) COVID-19 is, in the end, an endothelial disease. Eur Heart J 41:3038-3044
B

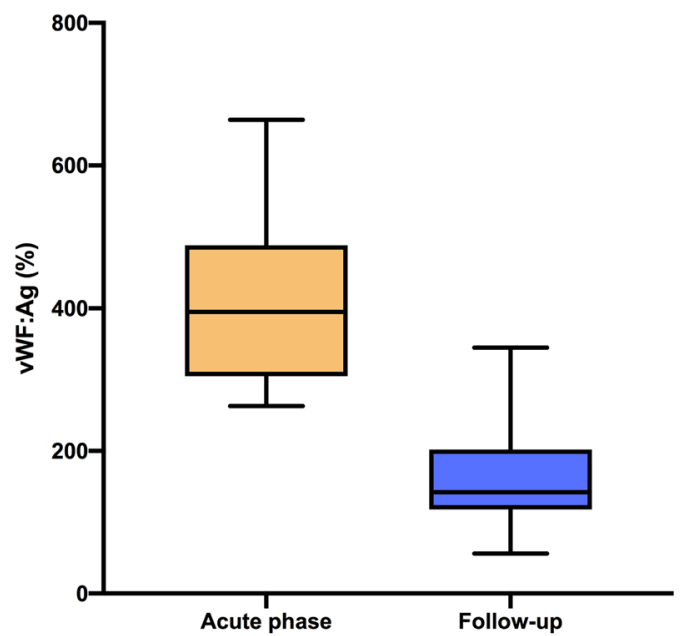

phase. At follow-up, blood samples were obtained at a median time of 144 days after COVID-19 diagnosis. COVID-19, coronavirus disease 2019; LA lupus anticoagulant, vWF:Ag, von Willebrand factor antigen

2. Bowles L, Platton S, Yartey N, Dave M, Lee K, Hart DP et al (2020) Lupus anticoagulant and abnormal coagulation tests in patients with Covid-19. N Engl J Med 383:288-290

3. Siguret V, Voicu S, Neuwirth M, Delrue M, Gayat E, Stépanian A et al (2020) Are antiphospholipid antibodies associated with thrombotic complications in critically ill COVID-19 patients? Thromb Res 195:74-76

4. Philippe A, Chocron R, Gendron N, Bory O, Beauvais A, Peron $\mathrm{N}$, et al. Circulating Von Willebrand factor and high molecular weight multimers as markers of endothelial injury predict COVID-19 in-hospital mortality. Angiogenesis 2021

5. Levine JS, Branch DW, Rauch J (2002) The antiphospholipid syndrome. N Engl J Med 346:752-763

6. Tang N, Li D, Wang X, Sun Z (2020) Abnormal coagulation parameters are associated with poor prognosis in patients with novel coronavirus pneumonia. J Thromb Haemost 18:844-847

7. Klok FA, Kruip MJHA, van der Meer NJM, Arbous MS, Gommers D, Kant KM et al (2020) Confirmation of the high cumulative incidence of thrombotic complications in critically ill ICU patients with COVID-19: An updated analysis. Thromb Res 191:148-150

8. Abdel-Wahab N, Lopez-Olivo MA, Pinto-Patarroyo GP, Suarez-Almazor ME (2016) Systematic review of case reports of antiphospholipid syndrome following infection. Lupus 25:1520-1531

Publisher's Note Springer Nature remains neutral with regard to jurisdictional claims in published maps and institutional affiliations. 